\title{
Characterization of Flavobacterium Species by Analysis of Volatile Fatty Acid Production
}

\author{
By D. RASOAMANANJARA, ${ }^{1}$ F. PELADAN, ${ }^{1}$ J. C. TURLOT, ${ }^{2}$ \\ H. MONTEIL ${ }^{*}$ AND C. RICHARD ${ }^{2}$ \\ ${ }^{1}$ Institut de Bactériologie de la Faculté de Médecine, Université Louis Pasteur, 3, rue Koeberlé, \\ 67000 Strasbourg, France \\ ${ }^{2}$ Centre d'Études Statistiques, IRMA, Université Louis Pasteur, 7, rue Descartes, \\ 67000 Strasbourg, France \\ ${ }^{3}$ Service des Entérobactéries, Unité INSERM 199, Institut Pasteur, \\ 75724 Paris Cedex 15, France
}

(Received 3 June 1986)

Seventy-four Flavobacterium strains were characterized by gas-liquid chromatographic analysis of volatile fatty acids produced in the culture medium. Principal components analysis permitted the graphic representation of the relative positions of the different strains, and aggregation according to the variance enabled a hierarchical classification to be established. The study revealed three subgroups each for $F$. meningosepticum and $F$. odoratum. Our $F$. breve, Flavobacterium sp. group IIb and $F$. multivorum strains appeared to be homogeneous. These results tallied with those of previous studies on DNA base composition and reassociation, electrophoretic protein profiles and cellular fatty acid composition.

\section{INTRODUCTION}

The genus Flavobacterium (Holmes et al., 1984) includes pigmented, strictly aerobic, nonmotile bacteria, with a DNA G $+\mathrm{C}$ content ranging from 30 to $42 \mathrm{~mol} \%$. Seven species are described by Holmes et al. (1984): $F$. aquatile, $F$. breve, $F$. balustinum, $F$. meningosepticum, $F$. odoratum, $F$. multivorum and $F$. spiritivorum. Fla vobacteria are common in soil and water and are frequently isolated in hospitals. In human pathology, the role of $F$. meningosepticum is well known and the unnamed Flavobacterium sp. group IIb of King (1959) is sometimes involved. Other species, such as $F$. odoratum, $F$. breve and $F$. multivorum, have also been implicated in nosocomial infections and have been isolated in liquids for hospital use and in wounds with secondary infections. Marked drug-resistance patterns of these bacteria have increased their clinical interest (Von Graevenitz, 1981). The identification of Flavobacterium by biochemical characters may be difficult. The present study was intended to develop a rapid method for characterizing some species of this genus by analysis of volatile fatty acids (VFA) produced in culture supernatants, a method that has proved useful for pseudomonads (Peladan \& Monteil, 1984 a; Peladan et al., 1984).

\section{METHODS}

Bacterial strains. Seventy-four strains were tested (Table 1): 31 F. meningosepticum, 9 F. breve, 13 Flavobacterium sp. group IIb, $15 \mathrm{~F}$. odoratum and $6 \mathrm{~F}$. multivorum. Ten were reference strains. Fifty-three were isolated at Strasbourg University Hospital and 11 were from Dr M. J. Pickett (Department of Microbiology, University of California, Los Angeles, Calif., USA). Table 1 gives the identities of strains as determined by Richard \& Monteil (1983). All strains were kept at $-80^{\circ} \mathrm{C}$ in a medium containing brain heart Infusion (Difco), horse serum and glycerol (10:1:1, by vol.).

Abbreviations: HAC, hierarchical ascendant clustering; PCA, principal components analysis; VFA, volatile fatty acids. 
Table 1. Origin of strains and groups after classification

Strain

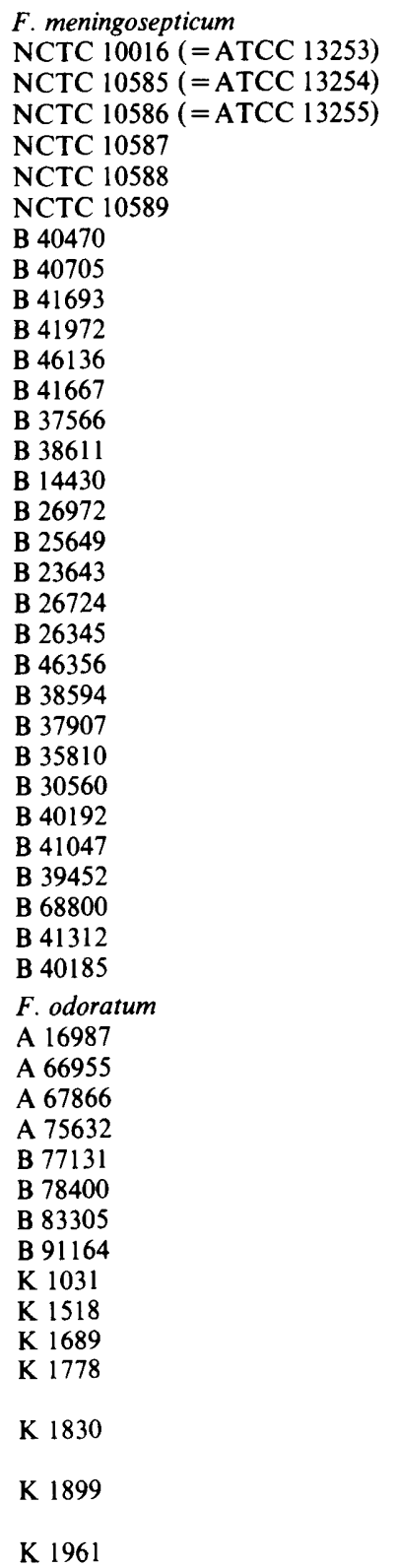

Flavobacterium sp. group IIb B 32502

B 37750

B 47466

B 47812

B 48260

B 49427

B 49942
Source

Serotype

Group after classification (see Fig. 1)
Reference strain

Reference strain

Reference strain

Reference strain

Reference strain

Reference strain

Bronchial secretion

Sputum

Sputum

Bronchial secretion

Sputum

Bronchial secretion

Sputum

Sputum

Catheter tip

Sputum

Aspiration fluid

Bronchial secretion

Bronchial secretion

Bronchial secretion

Bronchial secretion

Sputum

Blood

Bronchial secretion

Bronchial secretion

Bronchial secretion

Bronchial secretion

Bronchial secretion

Blood

Bronchial secretion

Bronchial secretion

Urine: Strasbourg

Urine: Strasbourg

Urine: Strasbourg

Urine: Strasbourg

Wound: Strasbourg

Bronchial secretion: Strasbourg

Ulcer: Strasbourg

Abcess: Strasbourg

St Mary's Hospital, Long Beach, Calif., 1975

API 76-2669, 1979

Clinical Laboratories, UCLA, Calif., 1980

Public Health Laboratories, Orange Country,

Calif., 1981

Huttington Memorial Hospital, Pasadena,

Calif., 1982

Wadsworth Veterans Administration

Hospital, Los Angeles, Calif., 1983

Biopathology Clinical Laboratories, North

Hollywood, Calif., 1983

Sputum
Sputum
Unknown
Unknown
Bronchial secretion
Unknown
Unknown

\begin{tabular}{|c|}
\hline $\mathbf{A}$ \\
\hline B \\
\hline$C$ \\
\hline D \\
\hline$E$ \\
\hline$F$ \\
\hline$G$ \\
\hline G \\
\hline$G$ \\
\hline$G$ \\
\hline$G$ \\
\hline G \\
\hline $\mathrm{G}$ \\
\hline$G$ \\
\hline$G$ \\
\hline$G$ \\
\hline \\
\hline$G$ \\
\hline$G$ \\
\hline G \\
\hline$G$ \\
\hline $\begin{array}{l}\mathrm{G} \\
\mathbf{F}\end{array}$ \\
\hline 1 \\
\hline
\end{tabular}

$\mathrm{X}$

C

F

A

A

A

A

$\mathrm{C}$

$\mathbf{F}$

C

F

A

C

$\mathrm{E}$
$\mathrm{E}$
$\mathrm{E}$
$\mathrm{E}$
$\mathrm{E}$
$\mathrm{E}$
$\mathrm{E}$ 
Table 1. (continued)

B 69546
B 72648
B 77095
B 77833
B 82341
B 82423
$F$. multivorum
NCTC 1103
NCTC 11034
K 1210
K 1213
K 1222
K 1231

F. breve
CL $200 / 75$
CL $666 / 76$
A 54615
B 33892
B 38674
B 44444
B 49835
B 54566
B 79158

Sputum

Sputum

Sputum

Sputum

Sputum

Sputum

Reference strain

Reference strain

API 76797, 1977 (Gilardi 2311)

API 76796, 1977 (Gilardi 2312)

API 76799, 1977 (Gilardi 2302)

California Department of Health, 13527 , Berkeley, Calif., 1977

Reference strain
Reference strain
Urine
Vaginal swab
Vaginal swab
Unknown
Unknown
Vaginal and anal swab
Unknown

E
E
E
E
E
E

H

H

H

H

$\mathrm{H}$

H

G

$\mathrm{X}$

G

G

G

G

G

G

G

Culture conditions. Starter cultures were prepared in $5 \mathrm{ml}$ trypticase soy broth (Diagnostics Pasteur, Paris) with shaking for $6 \mathrm{~h}$ at $30^{\circ} \mathrm{C} ; 1 \mathrm{ml}$ of this starter culture was added to $20 \mathrm{ml}$ of a culture medium comprising (per litre of medium): $\mathrm{CaCl}_{2}, 15 \mathrm{mg} ; \mathrm{MgSO}_{4} .7 \mathrm{H}_{2} \mathrm{O}, 123 \mathrm{mg} ; \mathrm{KH}_{2} \mathrm{PO}_{4}, 680 \mathrm{mg} ; \mathrm{K}_{2} \mathrm{HPO}_{4}, 2610 \mathrm{mg}$; Casamino acids, $20 \mathrm{~g}$; Ltryptophan, $10 \mathrm{mg} ; \mathrm{NaCl}, 1 \mathrm{~g}$; carbon source (glucose, sodium acetate and ethanol), each $1 \mathrm{~g}$; alcohols solution $\left(0.2 \mathrm{~g} \mathrm{l}^{-1}\right.$ of each of the following: $n$-propyl, $n$-butyl, isobutyl, $n$-pentyl and isopentyl alcohol), $1 \mathrm{ml}$; thiamin, $5 \mu \mathrm{g}$ (thiamin was the sole growth factor required for growth and optimal VFA production by all strains; the addition of other factors had no effect, or was inhibitory). The $\mathrm{pH}$ was adjusted, with $\mathrm{NaOH}$, to 7.5. Cultures were grown for $18 \mathrm{~h}$ at $30^{\circ} \mathrm{C}$ with shaking.

Extraction, identification and titration of VFA produced in culture supernatants. The methods of Peladan \& Monteil $(1984 b)$ were used. Peaks on the chromatograms were identified by comparing their retention times with those of chromatographically pure standards.

Data gathering. The values of the sterile control sample were subtracted from the chromatographic peak areas of the samples and the resulting values were normalized by expressing each acid produced as a percentage of the total acid output of the strain under examination.

Reproducibility of the method. Reproducibility was tested on six reference strains: two strains of $F$. meningosepticum (NCTC 10016, NCTC 10585), two of $F$. multivorum (NCTC 11033, NCTC 11034) and two of $F$. breve (CL 200/75, CL 666/76). Each strain was grown for two different periods in $20 \mathrm{ml}$ of two sets of culture medium inoculated with $1 \mathrm{ml}$ of the starter culture. Two extractions were performed on each culture supernatant and chromatographed three times, giving 24 chromatograms for each species tested. Variations were studied by computation of mean value and standard error for each acid and for each species.

Statistical analysis. Each strain is supposed to have a point representation on the set variables constituting a multidimensional space. Representation of all information obtained was achieved by means of the first factors of principal components analysis (PCA) (Harman, 1968; Hope, 1968; see Alderson, 1985, for a review). This representation was followed by a hierarchical ascendant clustering (HAC) classification based upon the same distances as for PCA, so the results of both methods could be compared.

Hierarchical classification. The distance between two strains in PCA is given by the usual Euclidian distance where the common variance of the variables is equal to 1 . HAC uses the same distance (similarity) to build the most homogeneous classes. The within-class variance defines the homogeneity of the classes. At each step, a new class is formed by amalgamation of the nearest two classes (Hartigan, 1975). The variance of the new class is greater than the sum of the variances of the two classes before grouping and the difference between these two quantities is called the level of clustering. This level reveals the similarity of the two classes. The results of the HAC are represented by a dendrogram. The selected partition can be interpreted on the graphs by the first principal components. 
Computing of results. SPAD programs edited by CESIA (Paris, France) were used for PCA and aggregation according to the variance and were run on UNIVAC 1110 computer at the Centre de Calcul du CNRS, Strasbourg, France.

\section{RESULTS}

We had originally intended to use data on the production of eight VFA for the analysis, but three of the VFA could not be used. The results for acetate were too variable for use; none of the strains produced any $n$-valeric acid, and the amounts of isocaproic acid produced were too small to be computed. The analysis was therefore based on the production of propionic, isobutyric, $n$ butyric, isovaleric and $n$-caproic acids.

Growth and VFA production kinetics of F. meningosepticum ATCC 10588 are shown in Fig. 1 (the other species gave similar results). An $18 \mathrm{~h}$ incubation was selected as the shortest time for enough growth and VFA production. Reproducibility of the methods is summarized in Table 2, showing mean acid percentages and $95 \%$ confidence limits for six reference strains. Variation was $<10 \%$ for each acid for all six strains. Typical chromatograms of the species are shown in Fig. 2. All six strains of $F$. multivorum produced a high proportion of isovaleric acid $(>80 \%)$ whereas strains of all the other species produced $<60 \%$.

The first three principal components accounted for $93 \%$ of the total variance, which was sufficient for the sample to be described. The correlations between variables and principal components are summarized in Table 3 and represented in Fig. 3. The first component, $F_{1}$, was characterized by isobutyric and isovaleric acids [correlation coefficients: $r\left(F_{1}, V_{2}\right)=+0.92$, $\left.r\left(F_{1}, V_{4}\right)=-0.99\right]$. Using these two variables, clusters $H$ and $C$ could be opposed to clusters $A$, $\mathrm{D}, \mathrm{E}$ and $\mathrm{F}$ (Fig. $3 a$ ). $\mathrm{F}_{2}$ gave the opposition between $n$-butyric and $n$-caproic acids (these two variables were uncorrelated with the others) and allowed differentiation of cluster $G$ from $B_{1}$ and $B_{2}$. Component $F_{3}$ was roughly defined by propionic acid and separated clusters $A, D$ and $E$ from $F$. The separation of the groups can be seen in Fig. 3. No variable distinguished groups $B_{1}$ and $\mathbf{B}_{2}$ correctly.

The results of the aggregation according to variance are summarized in Fig. 4. The hierarchical level at which clusters are delineated $(0 \cdot 12)$ was arbitrarily selected to give clusters corresponding approximately to phenetic species. Eight clusters were obtained: groups A to $\mathbf{H}$. Group A ( 7 strains) was composed of two subgroups: the first comprised 5 strains of $F$.odoratum (Table 1) and the second comprised $F$. odoratum A 16987 and $F$. breve CL 666/76. The latter subgroup (named group $X$ ) could not be considered as characteristic because of the small number of strains: this will be discussed below. Group B was composed of two $F$. meningosepticum subgroups: $B_{1}$ (9 strains) and $B_{2}$ (17 strains). However, these subgroups agglomerated at a low level. Group $C$ comprised 4 strains of $F$. odoratum, group D comprised 5 strains of $F$. meningosepticum, group E comprised 13 strains of Flavobacterium sp. group IIb, group F comprised 5 strains of $F$. odoratum, group $\mathrm{G}$ comprised 8 strains of $F$. breve, and group $\mathrm{H}$ comprised 6 strains of $F$. multivorum.

\section{DISCUSSION}

This study sought to establish a simple and rapid method for characterizing Flavobacterium. It should be borne in mind that the species we studied are represented by relatively few strains, and so the classification provided cannot be considered as definitive. Moreover, the level of cluster delineation in the dendrogram was arbitrarily selected so as to assign the strains to their original species. This level was not necessarily the best one, as the original species could have been split in unexpected ways. The representation of the strains on the first three principal axes of the PCA showed a good separation of the eight groups obtained by hierarchical clustering, and it also showed the variables that characterized these groups, especially isovaleric acid for group $\mathbf{H}$ and $n$-butyric acid for group G.

Flavobacterium sp. group IIb and $F$. multivorum were the most distinct taxa. As regards VFA production, Flavobacterium sp. group IIb (cluster group E) formed a single cluster, quite distinct from the others, especially from $F$. meningosepticum. This is valuable, since Richard \& Monteil 


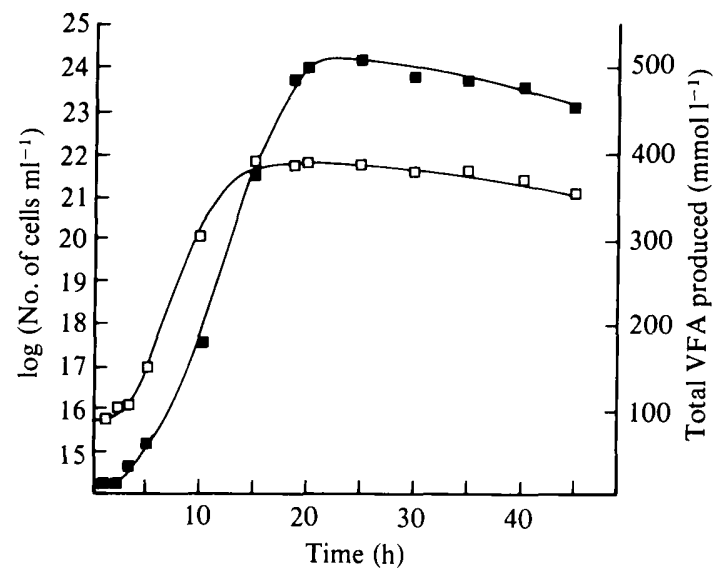

Fig. 1. Growth ( $\square$ ) and VFA production kinetics $(\square)$ of $F$. meningosepticum strain ATCC 10588.

Table 2. Mean values (percentage of total VFA) $\pm 95 \%$ confidence limits for $V F A$ produced by six reference strains

Strain

F. meningosepticum

NCTC 10016

$F$. meningosepticum

NCTC 10585

F. multivorum

NCTC 11033

F. multivorum

NCTC 11034

F. breve

CL 200/75

F. breve

CL 666/76

$$
\begin{aligned}
& \text { Propionic } \\
& \text { acid }
\end{aligned}
$$

$\begin{array}{ccc}8.9 \pm 0.72 & 66.1 \pm 4.8 & 0 \\ 22.5 \pm 1.7 & 55.5 \pm 4.21 & 0.48 \pm 0.03 \\ 1.7 \pm 0.09 & 9.91 \pm 0.52 & 1.61 \pm 0.12 \\ 1.72 \pm 0.1 & 6.0 \pm 0.32 & 1.51 \pm 0.1 \\ 20.2 \pm 2 & 48.2 \pm 4 & 13.7 \pm 0.05 \\ 18.9 \pm 0.52 & 47.2 \pm 3.5 & 6.7 \pm 0.6\end{array}$

Isovaleric acid

$22 \cdot 5 \pm 1 \cdot 2$

$22 \cdot 12 \pm 0 \cdot 18$

$84 \cdot 3 \pm 5 \cdot 2$

$92 \cdot 21 \pm 6 \cdot 31$

$16 \pm 1 \cdot 3$

$23 \cdot 82 \pm 1 \cdot 51$
n-Caproic
acid

$2 \cdot 8 \pm 0 \cdot 17$

$1.41 \pm 0.09$

$0 \cdot 33 \pm 0.02$

$0.53 \pm 0.04$

$0.1 \pm 0.01$

$0.43 \pm 0.02$

(1983) found that the only biochemical characters which separated $F$. meningosepticum from Flavobacterium sp. group IIb were amylase production and the intense pigmentation which became red after alkalinization. Owen \& Lapage (1974) found a similar DNA base composition and no consistent phenotypic differences between these two species, and their cellular fatty acid compositions are similar (Moss \& Dees, 1978). Our results do not indicate that Flavobacterium sp. group IIb is heterogeneous, as suggested by Owen \& Snell (1976) and Owen \& Holmes (1981), perhaps because of the small size of our sample. The study of more strains from various origins, and including reference strains, might have led to another conclusion.

VFA analysis showed that $F$. multivorum (group $\mathrm{H}$ ) was distinct from all other flavobacteria. Yabuuchi et al. (1983) reached the same conclusion, based on the presence of high concentrations of sphingolipids in the cellular lipid fraction. They placed $F$. multivorum in a new genus, Sphingobacterium, comprising $S$. spiritivorum, $S$. multivorum and $S$. mizutae.

In our study, $F$. odoratum, $F$. meningosepticum and $F$. breve showed differences from the accepted classification. The VFA analysis indicated three subgroups of $F$. odoratum. Although they considered this species as phenetically homogeneous, Owen \& Holmes (1978) showed that there were two DNA homology groups of $F$. odoratum which could be distinguished phenotypically only by their colony morphology. Furthermore, Owen \& Jackman (1983) showed, by means of electrophoretic protein patterns, that there were three distinct subgroups within $F$. odoratum, two of them corresponding to the DNA homology groups and a third 

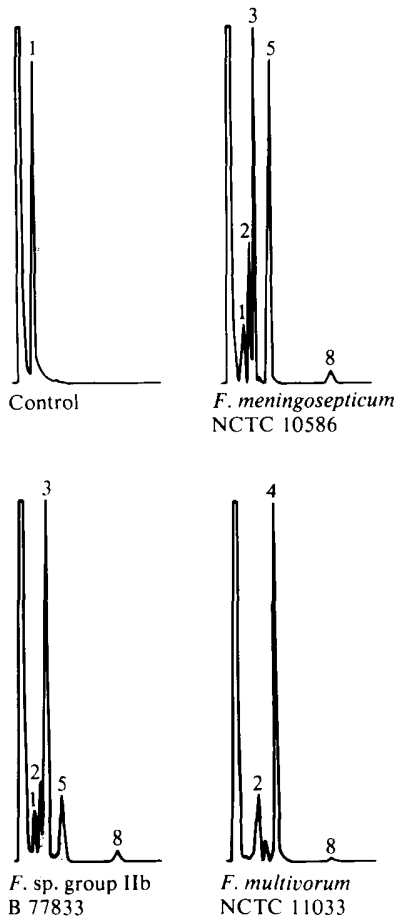
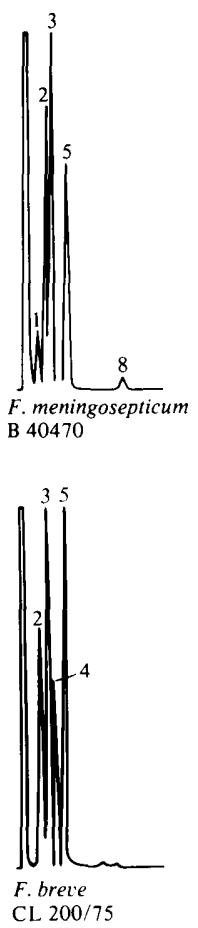
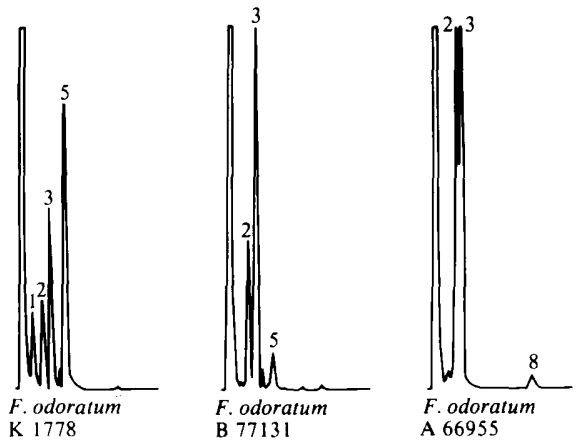

Fig. 2. Typical chromatographic profiles of five Flavobacterium species and the control (uninoculated medium). VFA: 1, acetic; 2 , propionic; 3, isobutyric; 4 , $n$-butyric; 5 , isovaleric; $6, n$-valeric; 7 , isocaproic; $8, n$ caproic.

intermediate group. It is not clear at present if the VFA groups correspond to the DNA homology groups.

Owen \& Lapage (1974) described $F$. meningosepticum as a biochemically homogeneous taxon, whereas Sottile et al. (1973) considered it heterogeneous on the basis of DNA reassociation, and suggested that most strains did not belong to the same species. This was confirmed by Holmes (1983) who reported some heterogeneity within $F$. meningosepticum. Our results appear to corroborate this: $F$. meningosepticum strains fell into two separate groups, B (consisting of subgroups $B_{1}$ and $B_{2}$ ) and $D$. Group D included all the reference strains except the type strain NCTC 10016. Owen \& Snell (1976) demonstrated that this strain was atypical on the basis of DNA reassociation. In our study, it clustered in the subgroup $B_{1}$ with lower similarity than all the other strains of this subgroup. All strains of group B except NCTC 10016 (serogroup A) and B 68800 (serogroup F) belonged to serogroup G. No biochemical tests allow differentiation of these groups.

Group G, comprising 8 of the $9 F$. breve strains studied, appeared to be rather homogeneous. The reference strain CL 666/76 clustered with $F$. odoratum A 16987 in group X, though it has been described as closely related to $F$. breve by DNA reassociation (Owen \& Holmes, 1980). We suggest two hypotheses to explain this. (1) This strain belonged to group G, which would imply that it is very widely scattered. This hypothesis would also be valid for $F$. odoratum group A which would be very scattered as well, there being no clear boundaries between the two groups. (2) This reference strain did not belong to group G, and although it was an authentic strain of $F$. breve, it belonged to another $F$. breve subgroup not examined in our study.

As regards group X, Fig. 3 shows that the centre of group $G$ is the closest to strain CL 666/76 and that of group A to strain A 16987.

In conclusion, this study has demonstrated that satisfactory characterization of the five Flavobacterium species studied can be achieved by analysis of five VFA. Quantitative results for VFA analyses are usually highly variable, but by normalizing the values, a maximum of $10 \%$ variability for each acid was obtained. Our results corroborate those of other studies, especially those based on DNA analysis. An important advantage of VFA analysis is its simplicity and 

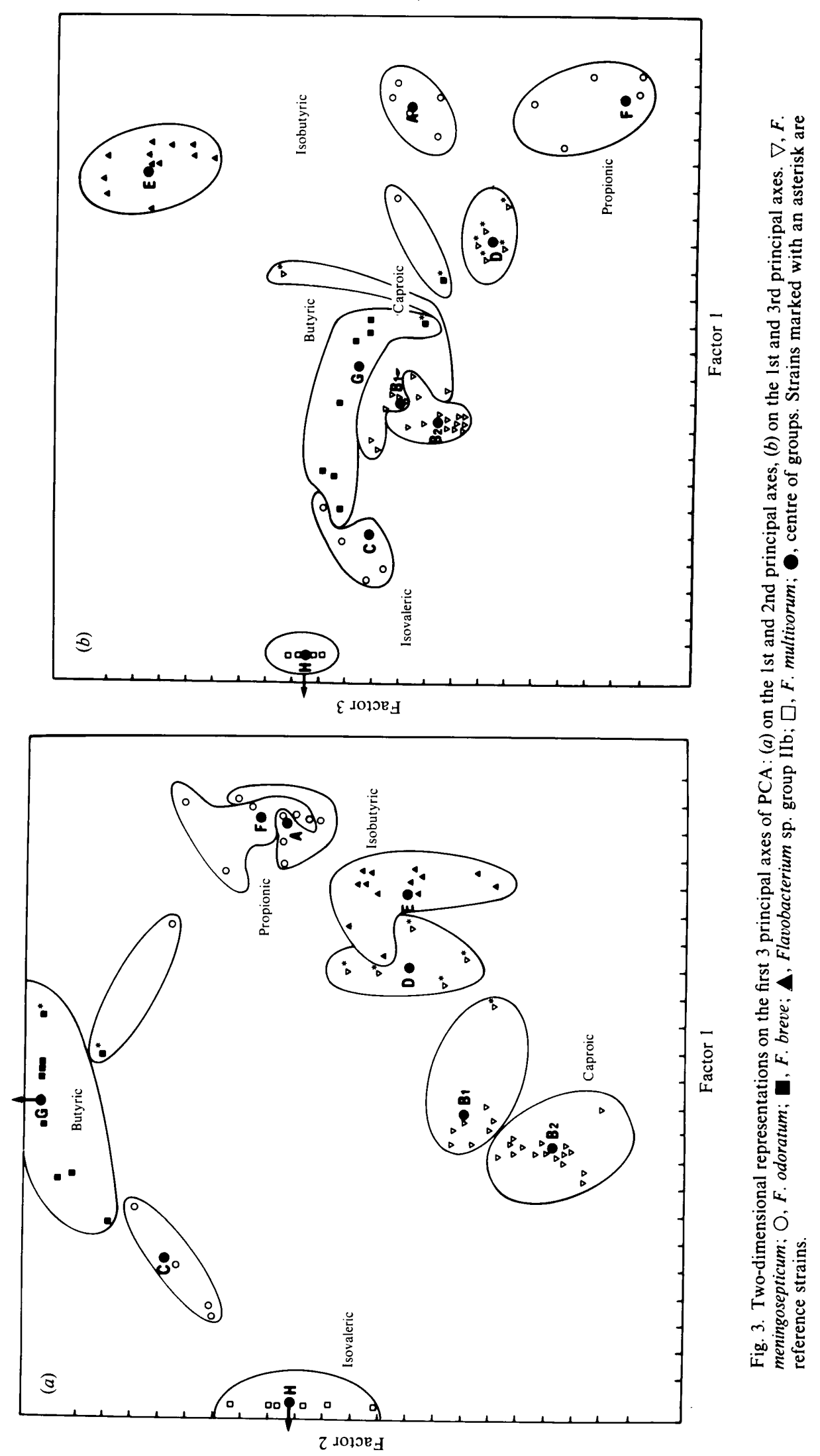


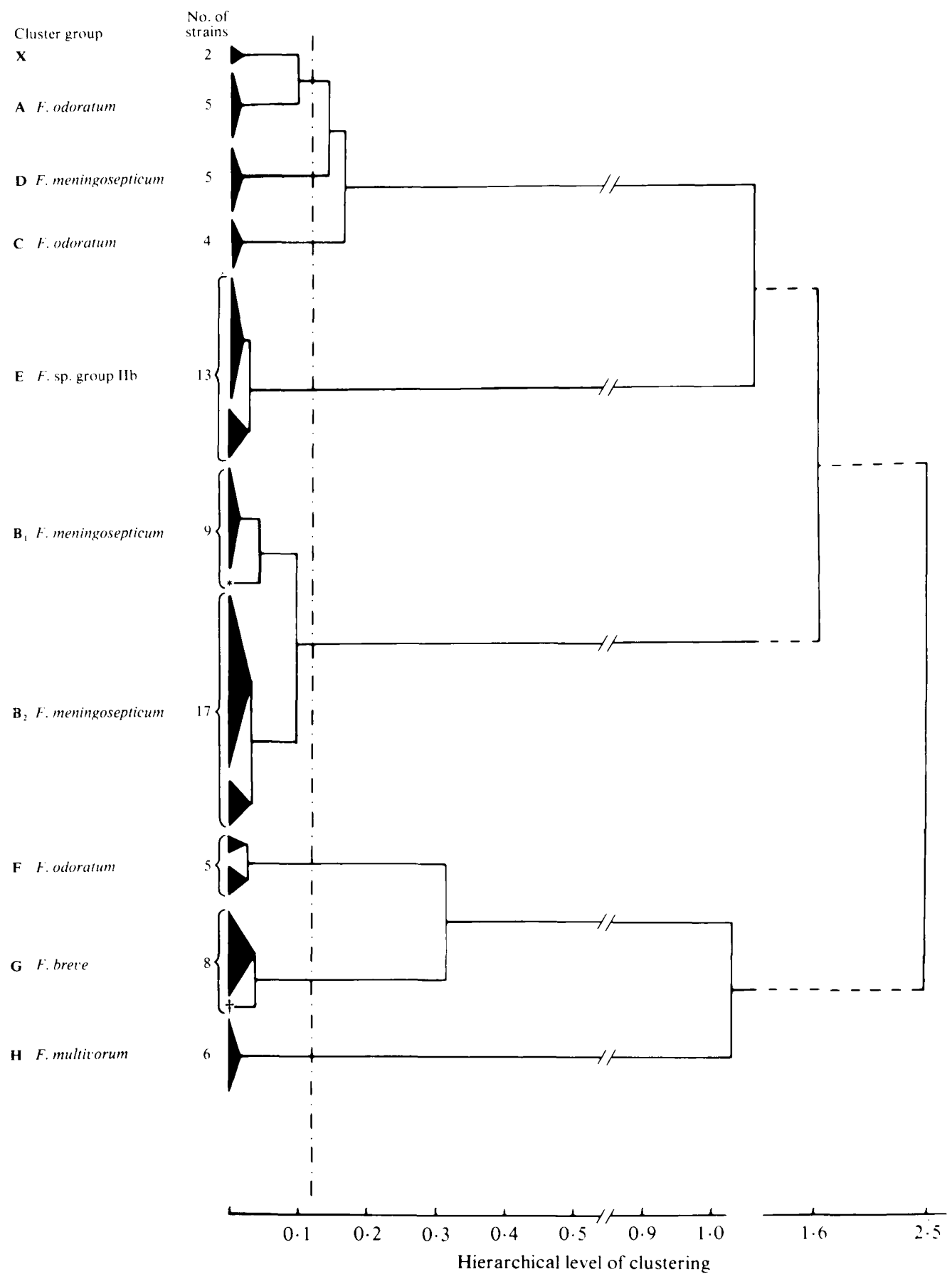

Fig. 4. Dendrogram of hierarchical classification of the Flavobacterium strains. The strains in each cluster are shown in Table 1. *, NCTC 10016; $\dagger$, B 49835.

speed. Once an organism has been assigned to the genus Flavobacterium, identification takes only $24 \mathrm{~h}(6 \mathrm{~h}$ starter culture, $18 \mathrm{~h}$ culture, $1 \mathrm{~h}$ extraction, $8 \mathrm{~min}$ chromatography), whereas 
Table 3. Correlation coefficients, $r$, between variables and principal components

\begin{tabular}{lrrr}
\multicolumn{1}{c}{ Variable } & \multicolumn{3}{c}{ Principal component: } \\
\cline { 2 - 4 } & \multicolumn{1}{c}{$\mathrm{F}_{1}$} & \multicolumn{1}{c}{$\mathrm{F}_{2}$} & \multicolumn{1}{c}{$\mathrm{F}_{3}$} \\
Propionic acid & 0.51 & 0.19 & -0.84 \\
Isobutyric acid & 0.92 & -0.06 & 0.40 \\
$n$-Butyric acid & -0.09 & 0.90 & 0.13 \\
Isovaleric acid & -0.99 & -0.10 & -0.09 \\
n-Caproic acid & 0.07 & -0.90 & -0.06
\end{tabular}

characterizing the various species by biochemical features takes 2 to $6 \mathrm{~d}$. We are currently developing a new method for identifying unknown strains, which does not involve PCA.

We are very grateful to Dr R. J. Pickett for providing some of the strains and to M. Ramboatina for helping in translation.

\section{REFERENCES}

ALDERSON, G. (1985). The application and relevance of non-hierarchic methods in bacterial taxonomy. In Computer-assisted Bacterial Systematics, pp. 227263. Edited by M. Goodfellow, D. Jones \& F. G. Priest. London: Academic Press.

HaRman, H. H. (1968). Modern Factor Analysis. Chicago \& London: University of Chicago Press.

Hartigan, J. A. (1975). Clustering Algorithms. New York: Wiley.

Holmes, B. (1983). The taxonomy of the genus Flavobacterium. In Gram-negative Bacteria of Medical and Public Health Importance: Taxonomy-Identification-Applications (Colloque Inserm no. 114). Edited by $\mathrm{H}$. Leclerc. Paris: Inserm.

Holmes, B., OWen, R. J. \& Hollis, G. (1982). Flavobacterium spiritivorum, a new species isolated from human clinical specimens. International Journal of Systematic Bacteriology 32, 157-165.

Holmes, B., Owen, R. J. \& MCMeekin, T. A. (1984). Genus Flavobacterium. In Bergey's Manual of Systematic Bacteriology, pp. 353-361. Edited by N. R. Krieg. Baltimore: Williams \& Wilkins.

HOPE, K. (1968). Methods of Multivariate Analysis. London: Unibooks.

KING, E. O. (1959). Studies on a group of previously unclassified bacteria associated with meningitis in infants. American Journal of Clinical Pathology 31, 241-247.

Moss, C. W. \& DeEs, S. B. (1978). Cellular fatty acids of Flavobacterium meningosepticum and Flavobacterium species group IIb. Journal of Clinical Microbiology 8, 772-774.

OWEN, R. J. \& Holmes, B. (1978). Heterogeneity in the characteristics of deoxyribonucleic acid from Flavobacterium odoratum. FEMS Microbiology Letters $\mathbf{4 8}$, 41-46.

OWen, R. J. \& Holmes, B. (1980). Differentiation between strains of Flavobacterium breve and allied bacteria by comparison of deoxyribonucleic acids. Current Microbiology 4, 7-11.

OWEN, R. J. \& Holmes, B. (1981). Identification and classification of Flavobacterium species from clinical sources. In Proceedings of the International Sympo- sium on Yellow-Pigmented Gram-Negative Bacteria of the Flavobacterium-Cytophaga Group, pp. 39-50. Edited by H. Reichenbach \& O. B. Weeks. Weinheim, Deerfield Beach \& Basel: Verlag Chemie.

OWEN, R. J. \& JACKMAN, P. J. H. (1983). Electrophoretic protein profiles of Flavobacterium odoratum. Newsletter of the Flavobacterium-Cytophaga Group 3, $10-12$.

OWEN, R. J. \& LAPAGE, S. (1974). A comparison of strains of King's Group IIb of Flavobacterium with Flavobacterium meningosepticum. Antonie van Leeuwenhoek 40, 255-264.

OWEN, R. J. \& SNELl, J. J. S. (1976). Deoxyribonucleic acid reassociation in the classification of Flavobacterium. Journal of General Microbiology 93, 89-102.

Peladan, F. \& Monteil, H. (1984a). Étude par chromatographie en phase gazeuse des acides gras volatils produits par quatorze espèces de Pseudomonas. Annales de microbiologie 135A, 399-410.

Peladan, F. \& MonteIl, H. (1984b). Intérêt taxonomique de la caractérisation des Pseudomonas par lanalyse des acides gras volatils produits en culture. Annales de microbiologie 135A, 411-425.

Peladan, F., Turlot, J. C. \& Monteil, H. (1984). Discriminant analysis of volatile fatty acids produced in culture medium: a novel approach to the identification of Pseudomonas species. Journal of General Microbiology 130, 3175-3182.

RichARD, C. \& MONTEIL, H. (1983). Isolement, identification et signification clinique des espèces du genre Flavobacterium. Annales de biologie clinique 41, $187-198$

Sottile, M. I., Baldwin, J. N. \& Weaver, R. E. (1973). Deoxyribonucleic acid hybridization studies on Flavobacterium meningosepticum. Applied Microbiology 26, 535-539.

Von GRAEVEnITZ, A. (1981). Clinical significance and antimicrobial susceptibility of flavobacteria. In Proceedings of the International Symposium on YellowPigmented Gram-Negative Bacteria of the Flavobacterium-Cytophaga Group, pp. 153-154. Edited by $\mathbf{H}$. Reichenbach \& O. B. Weeks. Weinheim, Deerfield Beach \& Basel: Verlag Chemie. 
Yabuuchi, E., Kaneko, T., Yano, I., Moss, C. W. \& MIYOSHI, N. (1983). Sphingobacterium gen. nov., Sphingobacterium spiritivorum comb. nov., Sphingobacterium multivorum comb. nov., Sphingobacterium mizutae sp. nov. and Flavobacterium indologenes $\mathrm{sp}$. nov. : glucose non-fermenting Gram-negative rods in CDC Group IIk-2 and IIb. International Journal of Systematic Bacteriology 33, 580-598. 